\title{
Multivariate Volatility Regulated Kelly Strategy: A Superior Choice in Low Correlated Portfolios
}

\author{
Ruanmin Cao1, Zhenya Liu ${ }^{2,3}{ }^{*}$, Shixuan Wang ${ }^{4}$, Weifeng Zhou ${ }^{2}$ \\ ${ }^{1}$ CITIC Securities Co., Ltd, Beijing, China \\ ${ }^{2}$ Economics department, University of Birmingham, Birmingham, UK \\ ${ }^{3}$ Finance Department, Renmin University of China, Beijing, China \\ ${ }^{4}$ Cardiff University, Cardiff, UK \\ Email: ^zhenya_liu@hotmail.com
}

How to cite this paper: Cao, R.M., Liu, Z.Y., Wang, S.X. and Zhou, W.F. (2017) Multivariate Volatility Regulated Kelly Strategy: A Superior Choice in Low Correlated Portfolios. Theoretical Economics Letters, 7, 1453-1472.

https://doi.org/10.4236/tel.2017.75098

Received: June 14, 2017

Accepted: August 12, 2017

Published: August 15, 2017

Copyright $\odot 2017$ by authors and Scientific Research Publishing Inc. This work is licensed under the Creative Commons Attribution International License (CC BY 4.0).

http://creativecommons.org/licenses/by/4.0/

\begin{abstract}
We propose a Multivariate Volatility Regulated Kelly strategy, which has extra penalization on variance compared to the Kelly criterion. The objective function is constructed and solved. We show the superiority of our method in relatively low correlated portfolios, relative to the fractional Kelly and full Kelly strategies. Our strategy reduces the short-term risk without sacrificing the growth rate to invest more in risk-free assets. Simulation results and Chinese commodity future empirical results strongly support our method.
\end{abstract}

\section{Keywords}

Kelly, Low Correlation, Portfolio

\section{Introduction}

Both the Kelly criterion and fractional Kelly strategies play important roles in asset allocation and portfolio investment. Early contributions to the theory and practice include Kelly [1], Latane [2], Breiman [3], Thorp [4] and Hakansson [5]. The Kelly criterion is widely applied in asset allocation [6] [7] [8]. Maclean, Thorp and Ziemba [9] conclude that there are both good and bad properties of the Kelly criterion. Its main advantage, which maximizes the expected value of the logarithm of wealth period by period, is that it maximizes the limiting exponential growth rate of wealth. The main disadvantage is that its suggested wagers may be very large. Hence, the Kelly criterion can be very risky in the short term.

To overcome high risk shortcoming, Ziemba [10] and Thorp [11] proposed the fractional Kelly strategy: invest a proportion $f$ of one's wealth in the Kelly 
portfolio and a proportion $1-f$ in the risk-free asset. There are two key benefits of the fractional Kelly strategy: first, the volatility of a fractional Kelly portfolio is significantly less than that of the full Kelly portfolio. Second, fractional Kelly strategies are optimal with assumptions of the Merton model [12]. In fact, theoretically, the fractional Kelly decreases volatility with a corresponding decrease in the long-run growth rate. The fractional Kelly is optimal in theory and often outperforms the full Kelly in empirical studies. Unfortunately, fractional Kelly strategies are no longer optimal when the log normality assumption is removed [13]. Many attempts have been made to retain the optimality of fractional Kelly strategies in recent years. Extensions of fractional Kelly-like risk sensitive benchmark and the Intertemporal Capital Asset Pricing Model (ICAPM) are used to guarantee optimality [14] [15].

In this paper, we propose a new direction for an improved Kelly strategy: a modified target function can outperform traditional Kelly or fractional Kelly strategies at both wealth growth and risk control side. Rather than maintaining the optimality of fractional Kelly strategies, we are more interested in seeking better risk estimators and giving inspiration in Kelly portfolio management. We call our approach the Multivariate Volatility Regulated Kelly (MVRK) strategy, which indeed provides a modified covariance estimator that is more adapted to low correlated portfolios. MVRK is inspired by time series momentum which assumes that asset correlation can be ignored [16] and is partly connected to the covariance shrinkage method [17].

Our results show that MVRK outperforms the full Kelly and fractional Kelly strategies in relatively low correlated portfolios. This is the main contribution of the paper. We have verified our findings in three ways. First, the theoretical intuition of MVRK is very clear and simple: volatility impacts on risk estimators are more important than correlation in low-correlated scenarios. Second, simulation results prove the advantage of MVRK in low correlation settings. Third, real data from the Chinese commodity market further support our method.

This paper is laid out as follows. In Section 2, we introduce the framework of Kelly portfolios and fractional Kelly portfolios in Merton assumptions. In Section 3, we show the methodology of the Multivariate Volatility Regulated Kelly strategy and explain the intuition compared with the full Kelly and fractional Kelly strategies. In Section 4, we simulate MVRK strategies and Kelly strategies with different correlation settings. Moreover, we show that MVRK is a better choice in low correlated portfolios. In Section 5, we use data from the Chinese commodity market and give empirical results with full Kelly, fractional Kelly and MVRK strategies. The empirical evidence further supports our method. In Section 6, we summarize the findings and make conclusions.

\section{The Kelly Criterion Portfolio and Fractional Kelly Strategies with Risk Sensitive Control}

\subsection{The Kelly Criterion Portfolio}

Assume we have a set of risky assets whose stochastic term is driven by $K$ 
wiener processes $W=\left(W_{1}, \cdots, W_{K}\right)^{\mathrm{T}}$, price dynamics then Figure

$$
\frac{\mathrm{d} S_{t}}{S_{t}}=\mu \mathrm{d} t+\Pi \mathrm{d} W
$$

where $S_{t}=\left(S_{1, t}, \cdots, S_{N, t}\right)^{\mathrm{T}}$ is price vector of $N$ assets at time $t . \mu$ is drift vector which has the same dimension of $N . \Pi$ is a $N \times K$ matrix, capturing the effect of different sources of uncertainty.

Similarly, considering a portfolio policy by investing $f=\left(f_{1}, \cdots, f_{K}\right)^{\mathrm{T}}$ in each asset, our portfolio thus follows SDE

$$
\frac{\mathrm{d} A_{t}}{A_{t}}=\left(1-f^{\mathrm{T}}\right) r \mathrm{~d} t+f^{\mathrm{T}} \mu \mathrm{d} t+\Pi \mathrm{d} W
$$

Apply Ito formula and define $N \times N$ covariance matrix $V=\Pi \Pi^{\mathrm{T}}$

$$
\mathrm{d} \log A_{t}=\left(r-f^{\mathrm{T}} r+f^{\mathrm{T}} \mu-\frac{1}{2} f^{\mathrm{T}} V f\right) \mathrm{d} t+\Pi \mathrm{d} W
$$

Let the drift term

$$
D=r-f^{\mathrm{T}} r+f^{\mathrm{T}} \mu-\frac{1}{2} f^{\mathrm{T}} V f
$$

The spirit of the Kelly system then involves maximizing $D$ with respect to $f$

$$
\frac{\partial D}{\partial f_{i}}=r-\mu_{i}+\sum_{j \neq i}^{N} \mu_{j}+v_{i, i} f_{i}=0
$$

Expand the portfolio to $N$ assets, and the first order condition will give

$$
f^{*}=(V)^{-1}(\mu-r \mathbb{I})
$$

where $\mathbb{I}$ is a $N \times N$ identity matrix. Substituting optimal $f^{*}$ back in (4), the optimal return growth rate is

$$
D\left(f^{*}\right)=r+\frac{(V)^{-1}(\mu-r \mathbb{I})^{2}}{2}
$$

This is the explicit solution to the multivariate Kelly criterion portfolio. It is clear that the Kelly criterion maximizes the geometric growth rate. It is also clear that extremely high weights of wealth may be allocated to some assets with very high independence (low correlation) across the portfolio. Diversification is one of the most important things to consider for a portfolio manager when constructing his/her portfolios. It is reasonable to accept the It is reasonable to accept the assumption that assets with low correlation will be added into the portfolio. The abnormal high weights on some assets contribute to high volatility in the short run. This is also a very crucial motivation for us to propose MVRK.

In case that no correlation exists and $K=N$, Equation (6) is simplified to

$$
f=(\operatorname{Diag}(V))^{-1}(\mu-r)
$$

Its implication is that an investor should invest any risky asset based solely on her observation on mean return and variance. This conclusion is consistent with univariate case. 


\subsection{Fractional Kelly Strategies with Risk Sensitive Control}

The fractional Kelly strategy is used to reduce this short-term risk and large wagers compared to the full Kelly strategy. We refer to betting less than Kelly as "fractional Kelly", which is simply a blend of Kelly and cash. However, this fractional Kelly is indeed a scaling of the full Kelly and reduces the risk at the expense of a lower growth rate.

We assume that asset prices are log normally distributed and that terminal portfolio wealth has a power utility function. When we introduce the relative risk aversion coefficient, the natural optimal solution from geometric Brownian motion is fractional Kelly policy. We apply Ito lemma with the power utility function $E\left(\frac{A^{1+\gamma}}{1+\gamma}\right)$, and this gives the optimal solution of the fractional Kelly.

From a fractional Kelly perspective, the risk sensitive asset model is the same as the fractional Kelly model [14] [15]. Intuitively, the objective function in fractional Kelly is to add risk sensitive control to drift term D in the Kelly portfolio previously derived:

$$
D-\frac{\gamma}{2} f^{\mathrm{T}} V f
$$

The explicit optimal solution to fractional Kelly will become

$$
f^{*}=\frac{1}{\gamma+1}(V)^{-1}(\mu-r \mathbb{I})
$$

with the relative risk sensitive coefficient $\gamma \in(-1, \infty)$.

Substituting (10) into $D-\frac{\gamma}{2} f^{\mathrm{T}} V f$, the optimal return growth rate is

$$
D\left(f^{*}\right)=r+\frac{(V)^{-1}(\mu-r \mathbb{I})^{2}}{2(\gamma+1)}
$$

From the derivation above, we notice that the release in power utility assumptions actually breaks down the optimality of the Kelly criterion strategies. The explicit solution is not optimal only considering maximum of the portfolio growth rate and final portfolio wealth. Comparing (6) and (10), fractional Kelly strategies adjust the weight of risky assets and geometric growth rate in the same size. Hence, the reduction in the risk is reflected in the corresponding reduction in growth rate.

\section{Multivariate Volatility Regulated Kelly}

In our recent study, we found that mean-variance risk control is not always a good choice. When we invest either in low correlated portfolios or in different asset classes with relatively low correlation, the penalty of assets' own variance becomes more important than covariance.

We use the same assumptions for the MVRK as for the Kelly criterion and fractional Kelly strategies: asset prices are log normal distributed and follow a geometric Brownian motion. In the fractional Kelly strategy, we change the 
objective function of portfolio wealth, adding an extra risk control term. In MVRK, we also add one extra volatility control term. We now change the objective function in the Kelly criterion to

$$
D-\frac{\theta}{2} f^{\mathrm{T}} \operatorname{Diag}(V) f
$$

$\theta \in(-1, \infty)$ is the volatility regulation coefficient. Then the explicit solution is

$$
f^{*}=(V+\theta \operatorname{Diag}(V))^{-1}(\mu-r \mathbb{I})
$$

Substituting (13) into (12), the growth rate of return now is:

$$
D\left(f^{*}\right)=r+\frac{(V+\theta \operatorname{Diag}(V))^{-1}(\mu-r \mathbb{I})^{2}}{2}
$$

Here $\operatorname{Diag}(V)$ is the diagonal line, which is idiosyncratic risk inherent in individual asset. Put another way, $\operatorname{Diag}(V)$ is volatility measure regardless of others' effect. The existence of the solution is explicit: $V+\theta \operatorname{Diag}(V)$ should not be degenerate. Note that $V$ is not invertible if $K<N$. This scenario can be true when we choose a redundant set. We call this "Multivariate Volatility Regulated Kelly" (MVRK). The implication of MVRK is clear: portfolio investment policy is restricted by an extra volatility regulation term and there is more aversion with regard to assets volatility and more weight put on the volatility regulation coefficient $\theta$.

On the one hand, MVRK does not scale the portfolio weight, and will not affect the risky assets return, which is apparently the problem of the fractional Kelly strategy. On the other hand, MVRK can avoid extremely high weights allocated to assets and dramatically reduce the short-term risk, which is presented as the main disadvantage of the Kelly strategy.

\section{Simulation}

\subsection{Simulation Assumptions and Settings}

In this section, we will show the simulation results of the MVRK and Kelly criterion strategies. We assume that the risk free rate is zero-the fractional Kelly is equivalent to the full Kelly under this setting. Hence, we only need to compare MVRK with the full Kelly strategy ${ }^{1}$. The relationship between the portfolio correlation and strategy performance is the main target. Portfolio size, trading length and the variation of the volatility regulation coefficient are also considered. We state several assumptions first.

Assumption 1: Correlated asset prices follow a geometric Brownian motion.

We allow assets to have correlations in the simulation. This is an important topic in quantitative finance, as it can be applied to simulating assets held in a portfolio that are dependent on one another to determine the underlying risk of

${ }^{1}$ When the risk free rate is positive, just scaling the same size of MVRK as for the fractional Kelly, we have a fractional MVRK. Comparing fractional MVRK and fractional Kelly strategies is always equivalent to comparing MVRK and full Kelly. As long as we show that MVRK can outperform full Kelly, we show that fractional MVRK can outperform fractional Kelly. 
the portfolio. A geometric Brownian motion model is used to keep consistent with the full Kelly, fractional Kelly and MVRK assumptions.

Assumption 2: Portfolio returns have zero mean but fluctuate slightly.

We set the portfolio average annual return to be equal to zero and allow some assets to have positive returns and others to have negative returns. In specific markets, asset annual returns are different, i.e. S \& P 500 has a 10\% annual return since its inception, while some futures markets can even have a negative annual return. In this paper, the major discussing point is risk estimators, so we suppose that we always invest in a zero mean portfolio.

Assumption 3: Asset returns have a fixed volatility level.

In practice, asset annual volatility can range from very low (less than $10 \%$ in bonds) to medium (ranging from $10 \%$ to $30 \%$ in equities) and to very high (more than $30 \%$ in commodities). We show simulation results for the MVRK and Kelly strategies with fixed $15 \%$ annual volatility. We fix the return and volatility level to eliminate any disturbance to correlation effects variation on strategies. Then we give some settings for the simulation.

Simulation Setting 1: Control correlation level $\rho_{i, j}$ from low 0.1 to 0.7 , with 0.1 step length.

Suppose we have a correlation matrix, denoted $C$.

$$
C=\left(\begin{array}{cccc}
1 & \rho_{1,1} & \cdots & \rho_{1, n} \\
\rho_{2,1} & 1 & \cdots & \rho_{2, n} \\
\vdots & \vdots & \ddots & \vdots \\
\rho_{n, 1} & \rho_{n, 2} & \cdots & 1
\end{array}\right)
$$

Simulation Setting 2: Control the portfolio dimension as 20,30 and 40.

Simulation Setting 3: Control trading length as 1250 trading days, 2500 trading days and 7500 trading days.

Simulation Setting 4: Simulation times $=1000$.

Simulation Setting 5: Set the fraction allocated in $\operatorname{Diag}(V)$ as $p=\frac{\theta}{1+\theta}$, and the fraction allocated in $V$ as $q=\frac{1}{1+\theta}$. We control the value of $p$ from 0 to 1 with 0.01 step length, adjusting the volatility regulation coefficient from 0 to $\infty$.

In the case where $p=0$, MVRK is equivalent to the full Kelly strategy which means there is no extra restriction on the idiosyncratic variance on each asset.

In the case where $p=1$, MVRK actually collapses to a time series momentum (TSMOM) strategy. In our empirical study, it is also valid to assert that time series momentum portfolios maximize the median fortune and geometric growth with the assumption of independence across assets.

If the cross-sectional correlation is not negligible, however, the TSMOM portfolio fails. In the futures market, it might be safer to accept heterogeneity of commodities, which are easily violated in the stock market. As an indication of further research, we may change the Kelly criterion to "Correlation Regulated Kelly" in highly correlated portfolios. In this scenario, we just range the volatility regulation coefficient $\theta$ in $(-1,0)$. 


\subsection{Simulation Results}

In our experiments, we examine the relative performance of the full Kelly and MVRK strategies. The nine simulations vary asset numbers with 20, 30 and 40, and observation numbers with 1250, 2500 and 7500 successively. We compare the Sharpe ratio and Value at Risk in the two methods and show the probability that the MVRK strategies have higher Sharpe ratio and higher Value at Risk. It is not necessary to give the absolute values of Sharpe ratio and Value at Risk. For one thing, the simulation assumptions naturally give uncontrollable random effects on the portfolio, and hence on the strategy performance. For another, the primary purpose of the simulation is to compare the full Kelly and MVRK in different correlation settings.

First, we display the results with $p=0.5$, while in this setting, the volatility regulation coefficient $\theta=1$, which is an intermediate intensity coefficient. Under this scenario, it is very clear to overlook the relationship between correlation and strategy performance.

Table 1 clearly reports the correlation effects on the Kelly strategy and MVRK strategy. The monotonous decrease in the winning probability of the MVRK strategies can be observed in both the Sharpe ratio and VaR, with an increasing correlation level from 0.1 to 0.7 . In different portfolio dimension and observation numbers, the monotone pattern always holds. When observation numbers increase from 1250 to 7500, the monotonous decrease in the winning probability function is more convex. Therefore, the correlation effects on the MVRK and full Kelly strategies become more significant when the observation number is larger. We fail to find significant effects of portfolio dimensions. MVRK strategies keep leading positions (winning probability exceeds 50\%) in all simulations where the correlation is less than 0.4. Hence, MVRK is superior in low correlated portfolios while Kelly is better in high correlated portfolios.

Actually, the monotonous decreasing pattern can be observed in different MVRK strategies. In another words, with different volatility regulation coefficient $\theta$, we can still show that MVRK strategies perform better in low correlated portfolios.

Figure 1 and Figure 2 plot the 3D-curves of the correlation effects on the Sharpe ratio and VaR winning probability that vary the fraction $p=\frac{\theta}{1+\theta}$ allocated in $\operatorname{Diag}(V)$ from 0.01 to 1 with a step of 0.01 . The monotonous decreasing pattern happens in most cases except for negligibly small $p$ (less than 0.1 ). The results are generally consistent with the $p=0.5$ results in Table 1 , and hence consistent with the conclusion in Table 1.

A very interesting finding, which is different from 20 and 30 dimension situations, is that in the 40 -dimension portfolio, the MVRK strategies present very low winning probabilities with high $p$ values even when the correlation is as small as 0.1 . Looking back to the case $p=1$, an MVRK strategy is equivalent to a TSMOM strategy and no correlation is considered at all. The sharply decreasing performance of the high-p-MVRK strategies even in low correlation implies 
20 dimension, 1250 observation number

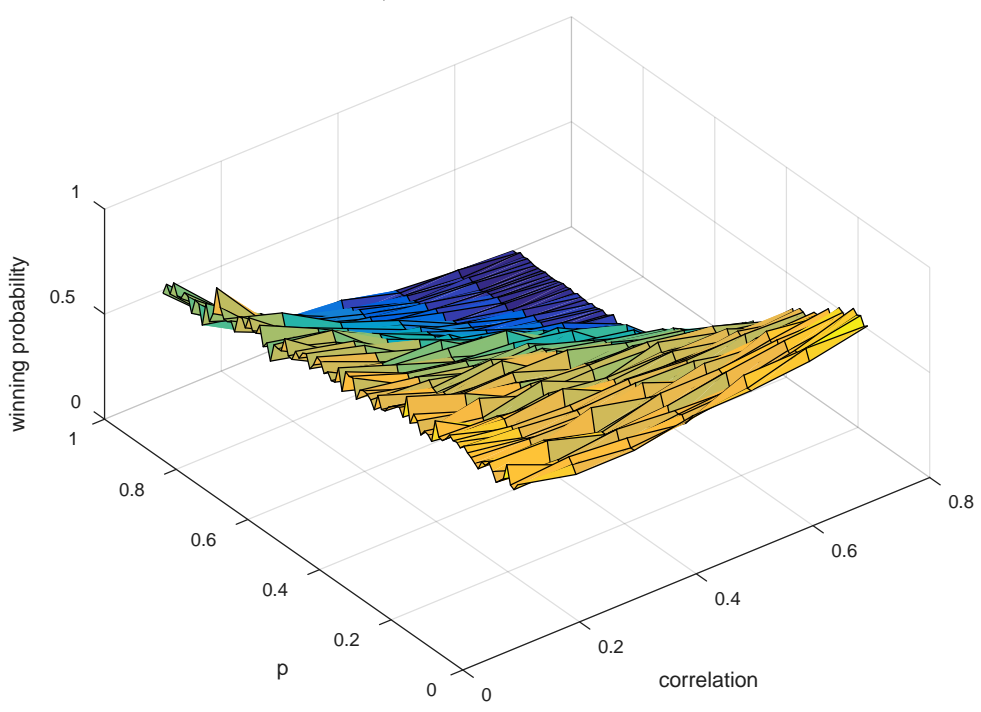

20 dimension, 2500 observation number

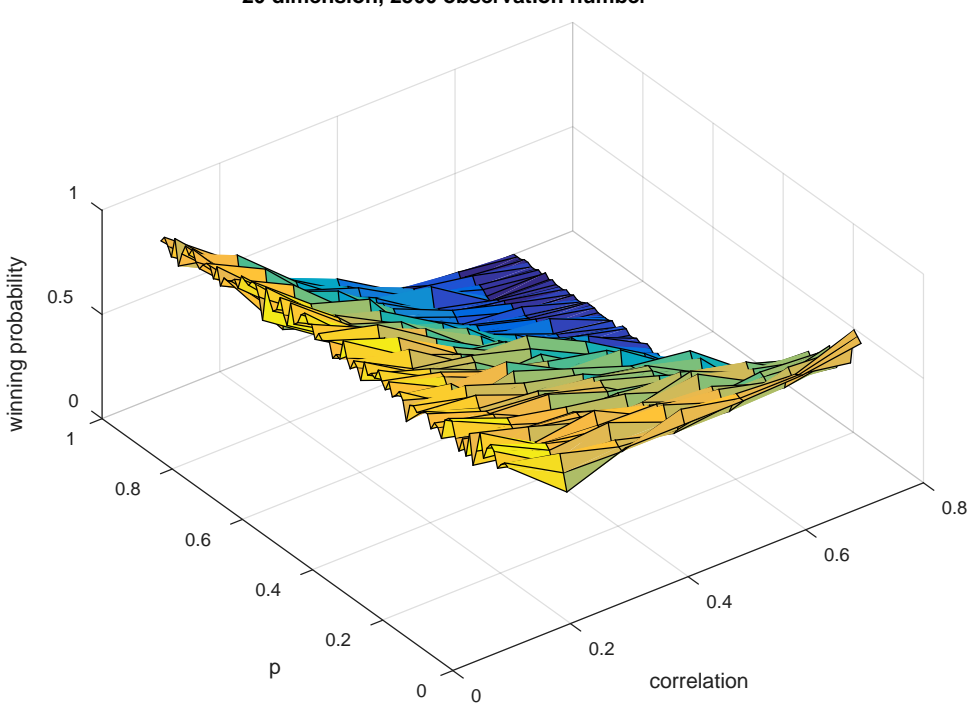

20 dimension, 7500 observation number

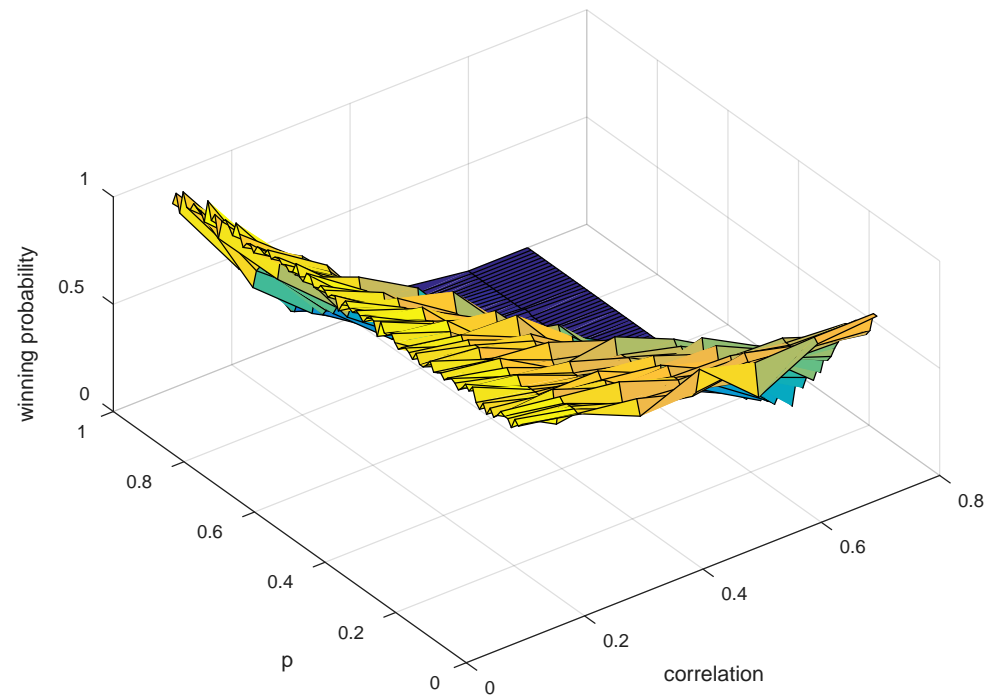



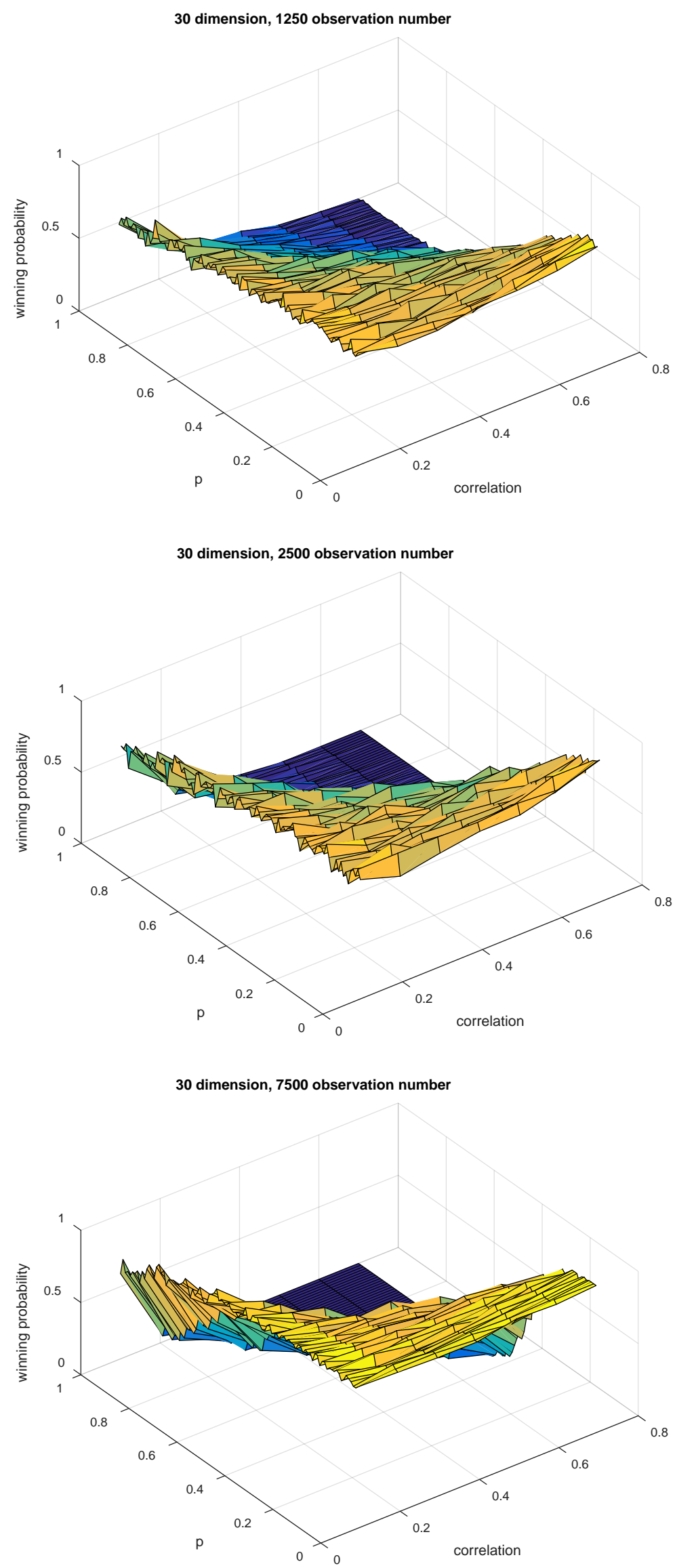

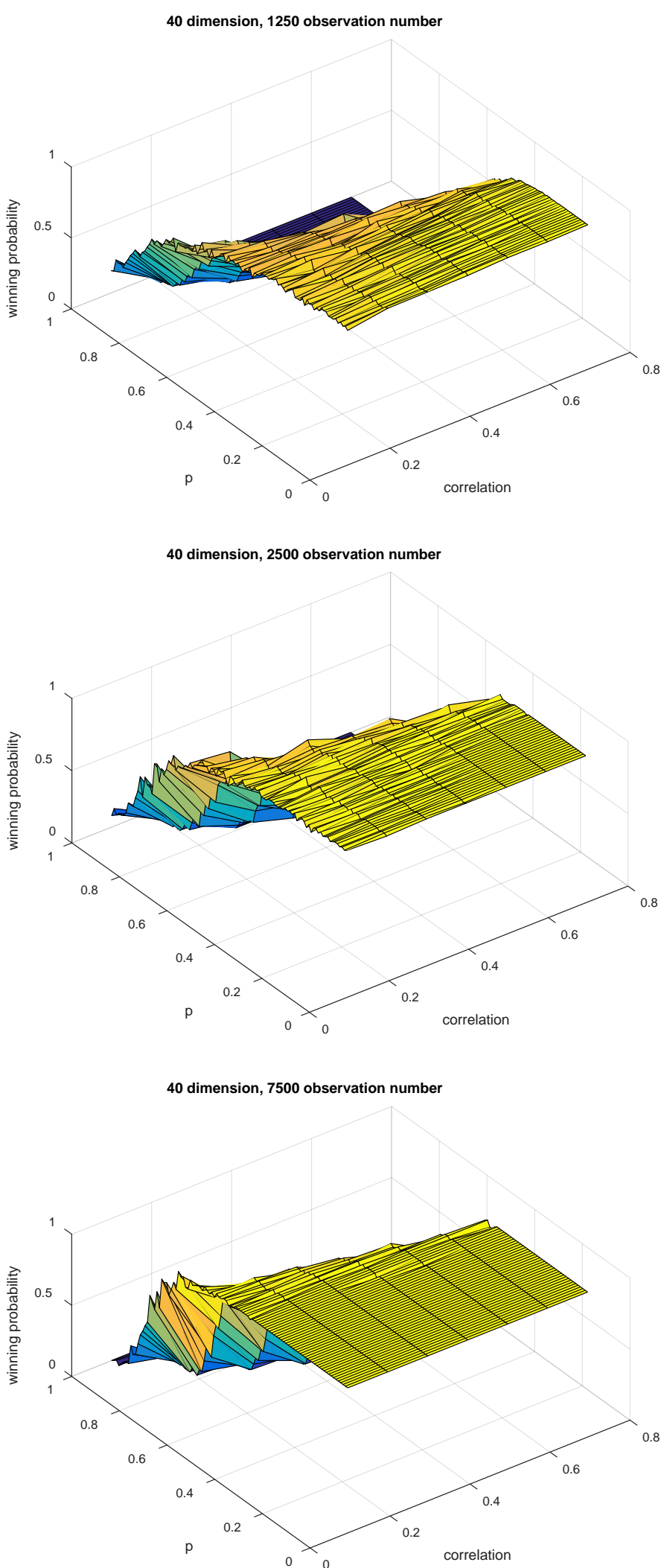

Figure 1. Fixed dimension and observation numbers, Sharpe ratio winning probability varying transformed volatility regulation coefficient $p$ from 0.01 to 1 . The lines along the $\mathrm{x}$-axis are winning probability curves in fixed $p$. The lines along the $\mathrm{y}$-axis are winning probability curves at a fixed correlation level. 

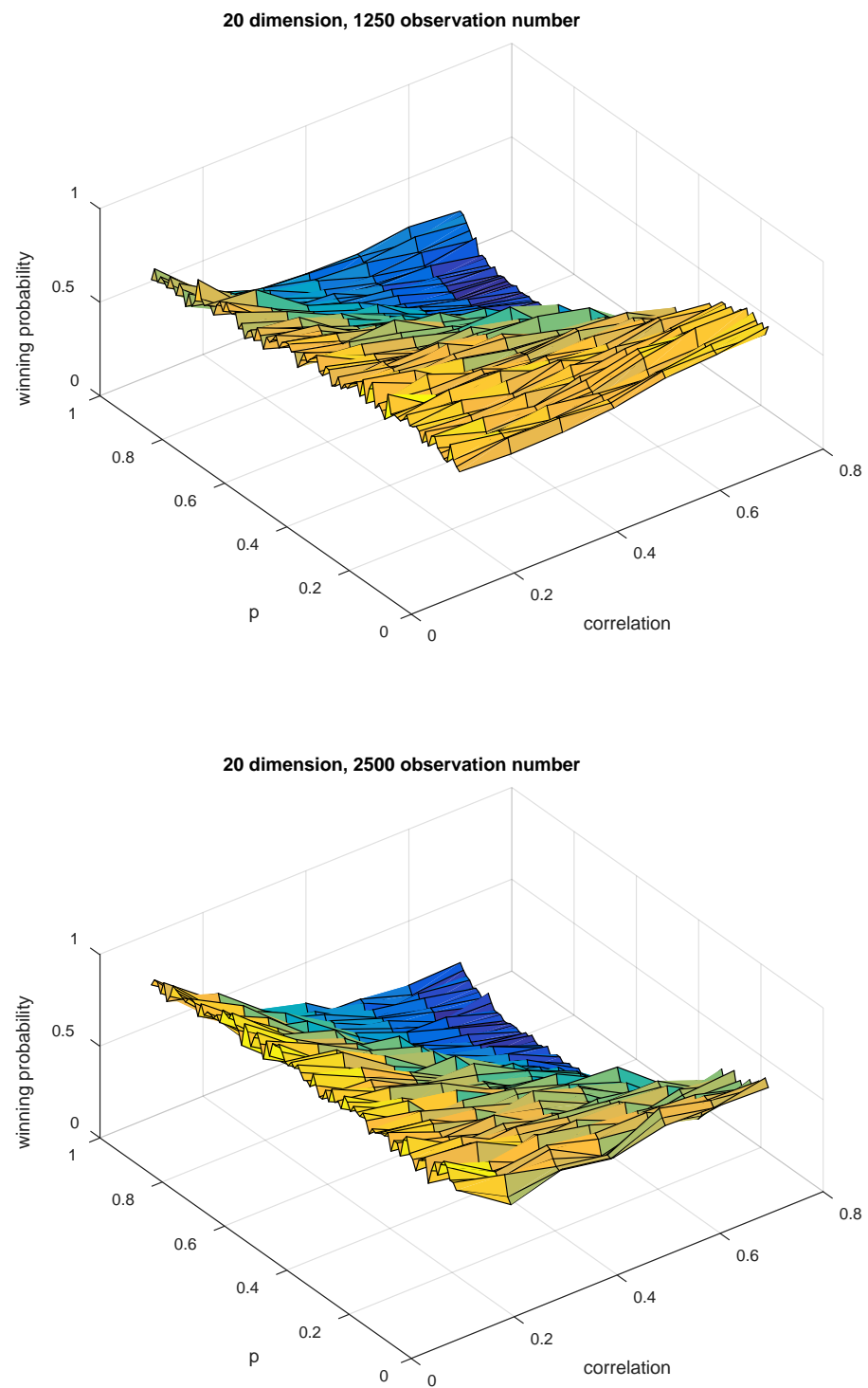

20 dimension, 7500 observation number

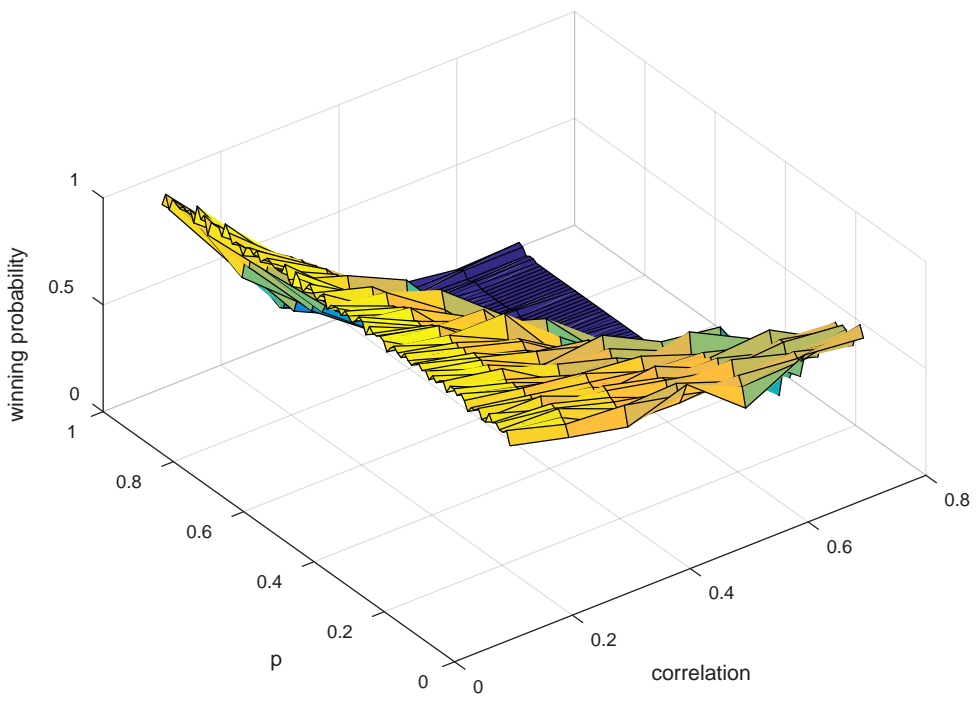




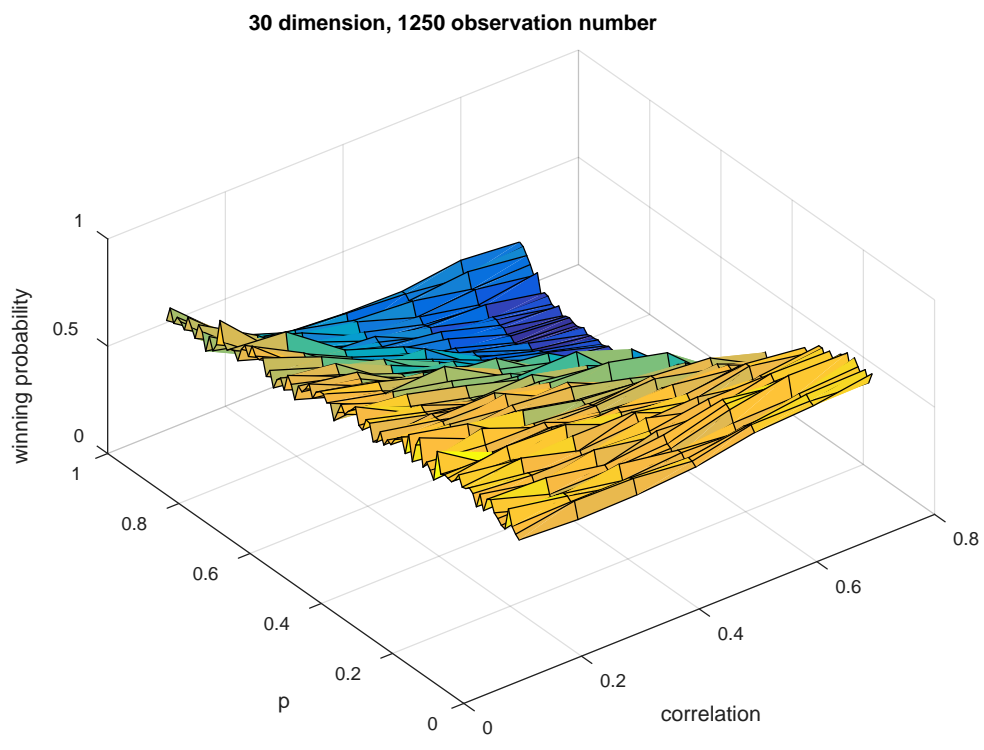

30 dimension, 2500 observation number

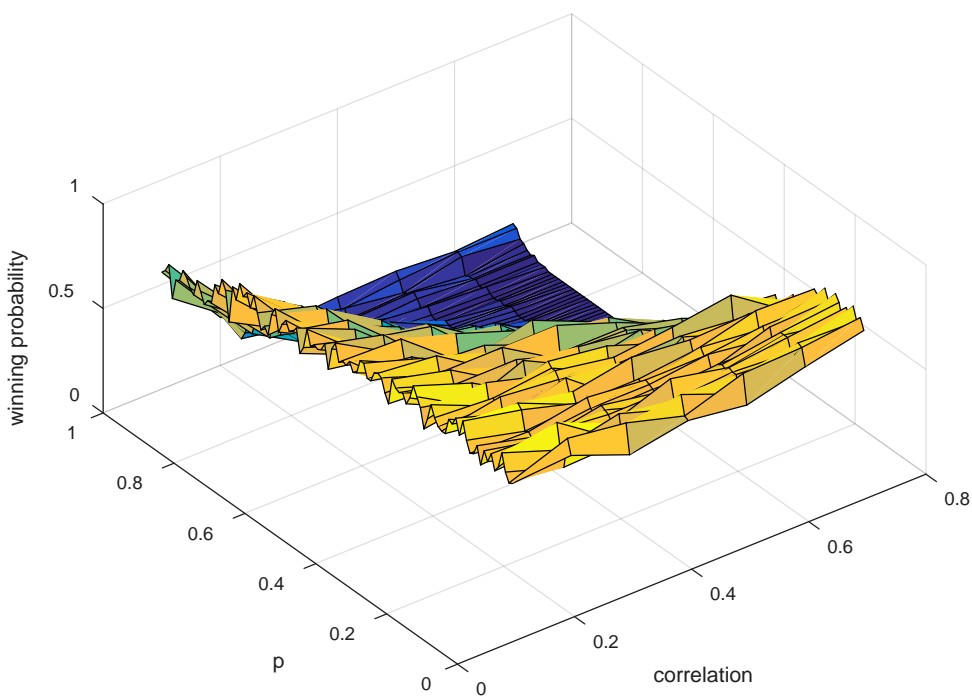

30 dimension, 7500 observation number

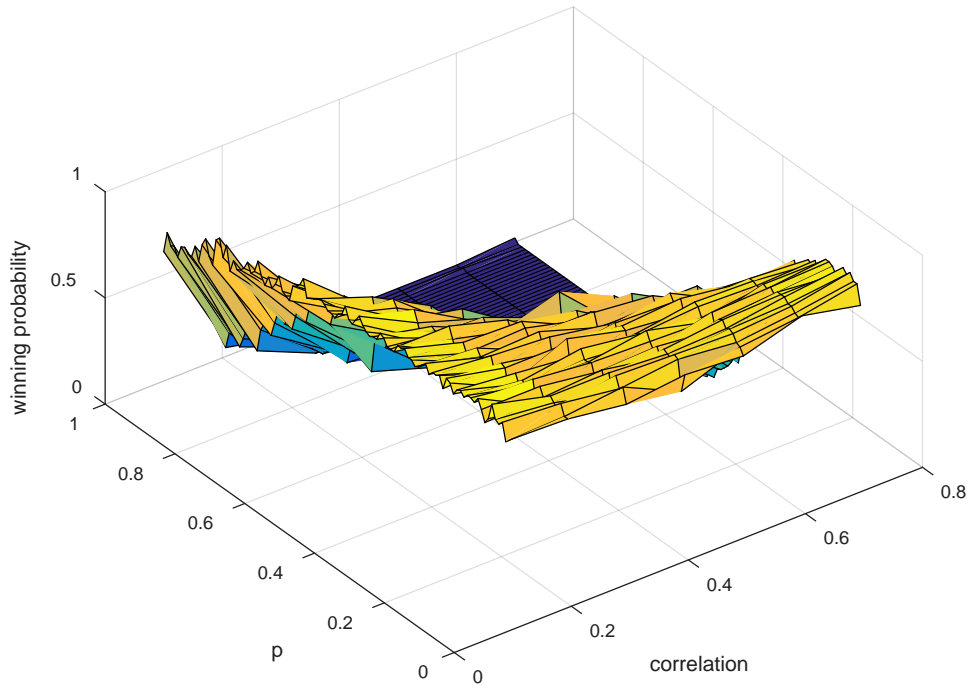



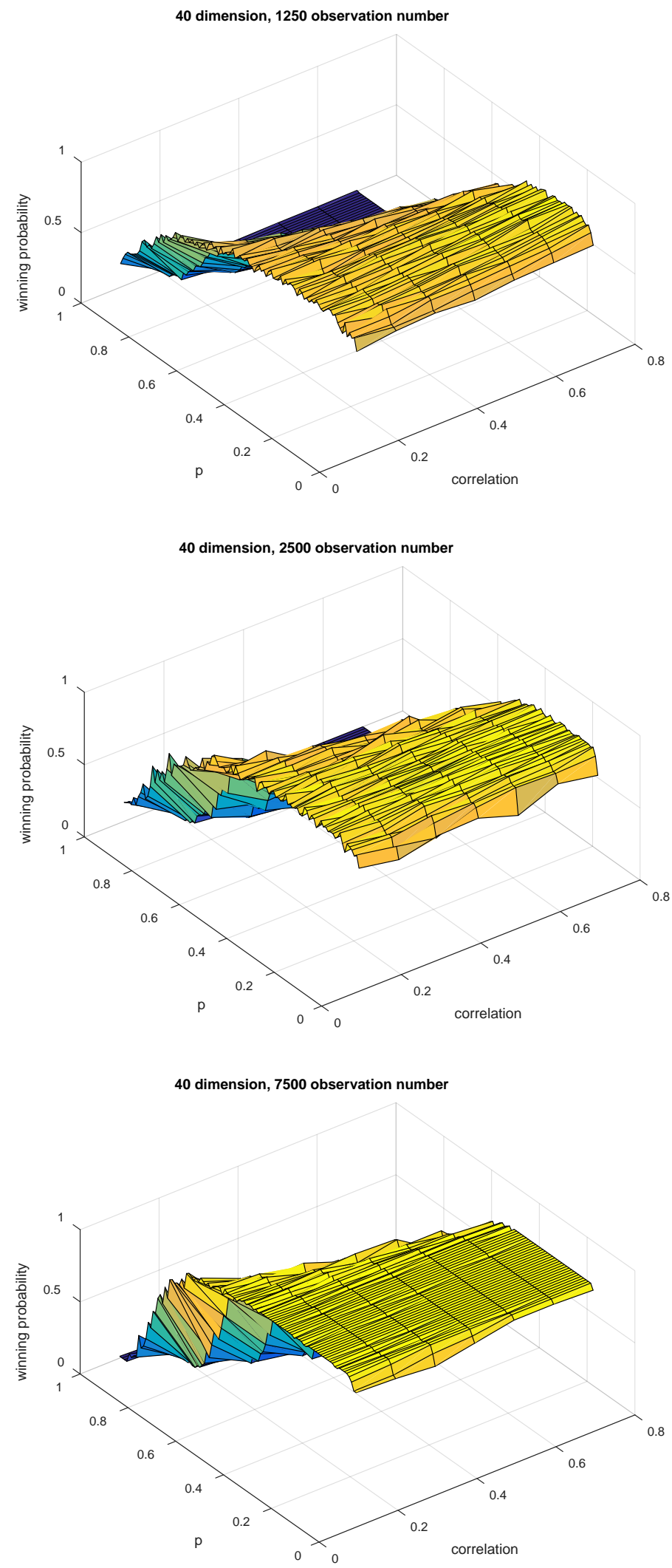

Figure 2. Fixed dimension and observation numbers, VaR winning probability varying transformed volatility regulation coefficient $p$ from 0.01 to 1 . The lines along the $\mathrm{x}$-axis are wining probability curves in fixed $p$. The lines along the $y$-axis are wining probability curves at a fixed correlation level. 
Table 1. Simulation results of MVRK and Kelly strategies. Correlation level of the portfolio varies from 0.1 to 0.7 . The table summarizes Sharperatio and VaR winning probability of MVRK strategies. $p=0.5$.

\begin{tabular}{|c|c|c|c|c|c|c|}
\hline Dimension: 20 & \multicolumn{2}{|c|}{ Obervation: 1250} & \multicolumn{2}{|c|}{ Obersvation: 2500} & \multicolumn{2}{|c|}{ Obersvation: 7500} \\
\hline Correlation/MVRK Win Prob & Sharpe ratio & $\mathrm{VaR}$ & Sharpe ratio & $\mathrm{VaR}$ & Sharpe ratio & $\mathrm{VaR}$ \\
\hline 0.1 & 0.74 & 0.73 & 0.83 & 0.82 & 0.97 & 0.96 \\
\hline 0.2 & 0.61 & 0.62 & 0.68 & 0.68 & 0.86 & 0.86 \\
\hline 0.3 & 0.54 & 0.54 & 0.58 & 0.60 & 0.70 & 0.72 \\
\hline 0.4 & 0.50 & 0.51 & 0.47 & 0.50 & 0.52 & 0.56 \\
\hline 0.5 & 0.38 & 0.40 & 0.35 & 0.39 & 0.30 & 0.36 \\
\hline 0.6 & 0.27 & 0.30 & 0.20 & 0.24 & 0.10 & 0.14 \\
\hline 0.7 & 0.15 & 0.19 & 0.07 & 0.10 & 0.001 & 0.01 \\
\hline Dimension: 30 & \multicolumn{2}{|c|}{ Obervation: 1250} & \multicolumn{2}{|c|}{ Obersvation: 2500} & \multicolumn{2}{|c|}{ Obersvation: 7500} \\
\hline Correlation/MVRK Win Prob & Sharpe ratio & $\mathrm{VaR}$ & Sharpe ratio & $\mathrm{VaR}$ & Sharpe ratio & $\mathrm{VaR}$ \\
\hline 0.1 & 0.70 & 0.69 & 0.77 & 0.78 & 0.89 & 0.88 \\
\hline 0.2 & 0.58 & 0.59 & 0.64 & 0.64 & 0.77 & 0.76 \\
\hline 0.3 & 0.54 & 0.51 & 0.55 & 0.56 & 0.62 & 0.62 \\
\hline 0.4 & 0.48 & 0.47 & 0.47 & 0.48 & 0.46 & 0.49 \\
\hline 0.5 & 0.40 & 0.40 & 0.35 & 0.37 & 0.29 & 0.31 \\
\hline 0.6 & 0.30 & 0.30 & 0.19 & 0.24 & 0.09 & 0.12 \\
\hline 0.7 & 0.13 & 0.17 & 0.04 & 0.08 & 0.002 & 0.01 \\
\hline Dimension : 40 & \multicolumn{2}{|c|}{ Obervation: 1250} & \multicolumn{2}{|c|}{ Obersvation: 2500} & \multicolumn{2}{|c|}{ Obersvation: 7500} \\
\hline Correlation/MVRK Win Prob & Sharpe ratio & $\mathrm{VaR}$ & Sharpe ratio & VaR & Sharpe ratio & $\mathrm{VaR}$ \\
\hline 0.1 & 0.69 & 0.68 & 0.75 & 0.75 & 0.90 & 0.90 \\
\hline 0.2 & 0.60 & 0.60 & 0.64 & 0.64 & 0.80 & 0.79 \\
\hline 0.3 & 0.57 & 0.58 & 0.61 & 0.60 & 0.74 & 0.74 \\
\hline 0.4 & 0.52 & 0.54 & 0.60 & 0.60 & 0.64 & 0.64 \\
\hline 0.5 & 0.50 & 0.53 & 0.49 & 0.50 & 0.51 & 0.53 \\
\hline 0.6 & 0.39 & 0.41 & 0.36 & 0.40 & 0.31 & 0.37 \\
\hline 0.7 & 0.29 & 0.31 & 0.17 & 0.22 & 0.04 & 0.09 \\
\hline
\end{tabular}

that it may be safer not to use pure TSMOM strategies in a portfolio including large numbers of assets. In contrast, when we have very small $p$ values, but with a high correlation, the MVRK strategies still can give higher winning probability in both Sharpe ratio and VaR. This phenomenon contrasts with our intuition: MVRK performs better in low correlated portfolios, while Kelly performs better in high correlated portfolios. However, it brings about a better risk estimator composed of a proper combination between variance and covariance estimators. The simulation results also reiterate the importance of the shrinkage method proposed by [17]. 


\section{Empirical Evidence from China Commodity Market}

\subsection{Data Set}

We use closing prices for 37 liquid China commodity futures since Chinese commodity market now has the largest trading volume [18]. The data spans the period from January 1, 2000 to July 11, 2016. The total sample size ranges from a low of 4 contracts at the beginning to a peak of 37 contracts from 2014.

The average pairwise correlation of return in our sample is about $26.5 \%$. We use interest rate published by the People's Bank of China as fixed risk free rates 2 . The summary statistics are provided in Table 2.

Table 2. Summary statistics on Chinese commodity futures contracts. The annualized mean returns and volatility (standard deviation) of the futures contracts are reported in our sample from January 2000 to July 2016.

\begin{tabular}{|c|c|c|c|c|c|}
\hline $\begin{array}{l}\text { Commodity } \\
\text { Futures Code }\end{array}$ & $\begin{array}{l}\text { Annualized } \\
\text { Return }\end{array}$ & $\begin{array}{l}\text { Annualized } \\
\text { Volatility }\end{array}$ & $\begin{array}{l}\text { Commodity } \\
\text { Futures Code }\end{array}$ & $\begin{array}{c}\text { Annualized } \\
\text { Return }\end{array}$ & $\begin{array}{c}\text { Annualized } \\
\text { Volatility }\end{array}$ \\
\hline & & & JD & $2.36 \%$ & $23.84 \%$ \\
\hline A & $3.63 \%$ & $17.99 \%$ & $\mathrm{JM}$ & $-18.17 \%$ & $20.68 \%$ \\
\hline AG & $-8.16 \%$ & $21.80 \%$ & $\mathrm{~L}$ & $-3.98 \%$ & $23.19 \%$ \\
\hline $\mathrm{AL}$ & $-2.21 \%$ & $13.41 \%$ & M & $2.70 \%$ & $20.45 \%$ \\
\hline $\mathrm{AU}$ & $3.41 \%$ & $18.71 \%$ & MA & $-11.53 \%$ & $19.89 \%$ \\
\hline B & $3.99 \%$ & $19.28 \%$ & NI & $-21.35 \%$ & $24.19 \%$ \\
\hline BB & $-10.52 \%$ & $32.69 \%$ & OI & $-4.27 \%$ & $18.94 \%$ \\
\hline $\mathrm{BU}$ & $-32.73 \%$ & $24.72 \%$ & $\mathrm{P}$ & $-6.68 \%$ & $22.33 \%$ \\
\hline C & $2.21 \%$ & $13.32 \%$ & $\mathrm{PP}$ & $-12.60 \%$ & $22.90 \%$ \\
\hline $\mathrm{CF}$ & $-0.06 \%$ & $16.85 \%$ & $\mathrm{RB}$ & $-5.74 \%$ & $17.51 \%$ \\
\hline CS & $-26.63 \%$ & $21.44 \%$ & $\mathrm{RM}$ & $1.67 \%$ & $21.47 \%$ \\
\hline $\mathrm{CU}$ & $1.18 \%$ & $21.09 \%$ & RU & $-1.84 \%$ & $25.02 \%$ \\
\hline $\mathrm{FB}$ & $-22.75 \%$ & $52.28 \%$ & SR & $2.06 \%$ & $18.98 \%$ \\
\hline FG & $-7.05 \%$ & $19.75 \%$ & TA & $-7.34 \%$ & $22.17 \%$ \\
\hline $\mathrm{FU}$ & $1.63 \%$ & $28.35 \%$ & $\mathrm{~V}$ & $-2.03 \%$ & $15.35 \%$ \\
\hline $\mathrm{HC}$ & $-11.39 \%$ & $19.59 \%$ & $\mathrm{WH}$ & $3.20 \%$ & $13.73 \%$ \\
\hline I & $-32.31 \%$ & $26.49 \%$ & $\mathrm{Y}$ & $1.82 \%$ & $19.04 \%$ \\
\hline IF & $-1.22 \%$ & $27.78 \%$ & $\mathrm{ZC}$ & $-10.14 \%$ & $15.93 \%$ \\
\hline $\mathrm{J}$ & $-17.68 \%$ & $19.98 \%$ & $\mathrm{ZN}$ & $-6.49 \%$ & $22.92 \%$ \\
\hline
\end{tabular}

${ }^{2}$ We compute the daily excess return of the most liquid futures contract (typically the nearest or next nearest-to-delivery contract), and then compound the daily returns to a continuous return index from which we can compute returns at any horizon. Then we calculate the annualized return and volatility based on the continuous return index. The risk free rate published by the People's Bank of China (PBC or PBOC) is available at

http://www.global-rates.com/interest-rates/central-banks/central-bank-china/pbc-interest-rate.aspx 


\subsection{Strategy Construction}

From (6), (10) and (13), investors can make portfolio decisions based on full Kelly, fractional Kelly and MVRK strategies respectively. We use past returns and calculate realized variance-covariance. Investors themselves can decide lookback periods. In our empirical study, we compare full Kelly strategies vs. MVRK strategies and fractional Kelly strategies vs. fractional MVRK strategies. Relative risk sensitive coefficient $\gamma$ and volatility regulation coefficient $\theta$ vary from zero to positive infinity. We keep simulation setting 5 for MVRK, but change the step length to 0.1. Similarly, in fractional Kelly strategies, we set the fraction allocated to risky assets as $m=\frac{\gamma}{1+\gamma}$, and the fraction allocated to riskfree assets as $n=\frac{1}{1+\gamma}$. We build long-short strategies by introducing an additional weight constraint:

$$
\left|f_{1}\right|+\left|f_{2}\right|+\left|f_{3}\right|+\cdots+\left|f_{n}\right|=1
$$

We compare the annual return, Sharpe ratio, maximum drawdown, 5\% value at risk and conditional $5 \%$ value at risk for these four strategies.

From Table 3, as the value of $p$ increases from 0.1 to 1.0 , the volatility regulation coefficient increases from very small to very big. Even the modest MVRK strategy with $p=0.1$ has a greater Sharpe ratio $(1.44>1.01)$ and a smaller maximum drawdown $(8.50 \%<8.65 \%)$ compared to the full Kelly strategy. When $p=0.7$, the MVRK strategy has the highest Sharpe ratio of 1.85 and a double annual return compared to the full Kelly strategy, with only $20 \%$ more maximum drawdown and value at risk. Moreover, if we scale the annual return to the same level, the MVRK strategies also show a better VaR and cVaR. In all other scenarios, MVRK strategies keep dominating superiority to the full Kelly strategy. Therefore, MVRK strategies always beat the full Kelly strategy in Chinese commodity markets.

The fractional Kelly strategies have a monotonously decreasing annual return and maximum drawdown when $\mathrm{m}$ is increasing, indicating that more weight is transferred from risky to risk free assets and that the portfolio becomes less risky at the expense of less wealth growth. Scaling the annual return to the same level for the fractional MVRK and fractional Kelly strategies, the statistics always show a higher return and a lower risk for the MVRK strategies. Even when $p \neq m$, the MVRK strategies always have a superior risk adjusted return compared to the fractional Kelly strategies. Hence, the MVRK strategies always beat the fractional strategies in Chinese commodity markets. An example with $p=m=0.5$, the same risk adjusted strategy performance is given in Figure 3.

In our sample of Chinese commodity markets, the average pairwise correlation level is $22.7 \%$, implying a relatively low correlated market. The empirical results significantly show that MVRK outperforms full Kelly and fractional Kelly in relatively low correlated portfolios. 
Table 3. Statistic summary of MVRK, Full Kelly, Fractional MVRK and Fractional Kelly strategies performance. The transaction cost is set as five in ten thousand per unit. $p=\frac{\theta}{1+\theta}$ is the fraction allocated $\operatorname{Diag}(\mathrm{V})$ and $m=\frac{\gamma}{1+\gamma}$ is the fraction allocated in the full Kelly strategy.

\begin{tabular}{|c|c|c|c|c|c|}
\hline Transaction cost. $0.05 \%$ & MVRK & $\begin{array}{l}\text { Full } \\
\text { Kelly }\end{array}$ & $\begin{array}{c}\text { Fractional } \\
\text { MVRK }\end{array}$ & $\begin{array}{c}\text { Fractional } \\
\text { Kelly }\end{array}$ & $p=m$ \\
\hline Annual return & $13.86 \%$ & $10.34 \%$ & $12.90 \%$ & $9.73 \%$ & 0.1 \\
\hline Sharpe ratio & 1.44 & 1.01 & 1.43 & 1.01 & \\
\hline Maximum Drawdown & $8.50 \%$ & $8.65 \%$ & $7.04 \%$ & $7.43 \%$ & \\
\hline $5 \% \mathrm{VaR}$ & $-0.51 \%$ & $-0.44 \%$ & $-0.45 \%$ & $-0.40 \%$ & \\
\hline cVaR & $-0.82 \%$ & $-0.73 \%$ & $-0.73 \%$ & $-0.65 \%$ & \\
\hline Annual return & $15.61 \%$ & $10.34 \%$ & $13.34 \%$ & $9.13 \%$ & 0.2 \\
\hline Sharpe ratio & 1.60 & 1.01 & 1.60 & 1.01 & \\
\hline Maximum Drawdown & $8.35 \%$ & $8.65 \%$ & $5.83 \%$ & $6.21 \%$ & \\
\hline $5 \% \mathrm{VaR}$ & $-0.53 \%$ & $-0.44 \%$ & $-0.42 \%$ & $-0.35 \%$ & \\
\hline $\mathrm{cVaR}$ & $-0.87 \%$ & $-0.73 \%$ & $-0.69 \%$ & $-0.58 \%$ & \\
\hline Annual return & $16.79 \%$ & $10.34 \%$ & $13.04 \%$ & $8.52 \%$ & 0.3 \\
\hline Sharpe ratio & 1.69 & 1.01 & 1.69 & 1.01 & \\
\hline Maximum Drawdown & $8.10 \%$ & $8.65 \%$ & $5.13 \%$ & $5.00 \%$ & \\
\hline $5 \% \mathrm{VaR}$ & $-0.56 \%$ & $-0.44 \%$ & $-0.39 \%$ & $-0.30 \%$ & \\
\hline $\mathrm{cVaR}$ & $-0.91 \%$ & $-0.73 \%$ & $-0.63 \%$ & $-0.50 \%$ & \\
\hline Annual return & $17.75 \%$ & $10.34 \%$ & $12.36 \%$ & $7.92 \%$ & 0.4 \\
\hline Sharpe ratio & 1.76 & 1.01 & 1.75 & 1.00 & \\
\hline Maximum Drawdown & $8.43 \%$ & $8.65 \%$ & $4.35 \%$ & $3.87 \%$ & \\
\hline $5 \% \mathrm{VaR}$ & $-0.60 \%$ & $-0.44 \%$ & $-0.35 \%$ & $-0.26 \%$ & \\
\hline $\mathrm{cVaR}$ & $-0.95 \%$ & $-0.73 \%$ & $-0.56 \%$ & $-0.43 \%$ & \\
\hline Annual return & $18.57 \%$ & $10.34 \%$ & $11.43 \%$ & $7.31 \%$ & 0.5 \\
\hline Sharpe ratio & 1.80 & 1.01 & 1.79 & 1.00 & \\
\hline Maximum Drawdown & $8.89 \%$ & $8.65 \%$ & $3.60 \%$ & $3.15 \%$ & \\
\hline $5 \% \mathrm{VaR}$ & $-0.62 \%$ & $-0.44 \%$ & $-0.30 \%$ & $-0.21 \%$ & \\
\hline $\mathrm{cVaR}$ & $-0.98 \%$ & $-0.73 \%$ & $-0.48 \%$ & $-0.36 \%$ & \\
\hline Annual return & $19.35 \%$ & $10.34 \%$ & $10.31 \%$ & $6.71 \%$ & 0.6 \\
\hline Sharpe ratio & 1.84 & 1.01 & 1.82 & 0.99 & \\
\hline Maximum Drawdown & $9.48 \%$ & $8.65 \%$ & $2.84 \%$ & $2.42 \%$ & \\
\hline $5 \% \mathrm{VaR}$ & $-0.63 \%$ & $-0.44 \%$ & $-0.24 \%$ & $-0.17 \%$ & \\
\hline $\mathrm{cVaR}$ & $-1.01 \%$ & $-0.73 \%$ & $-0.40 \%$ & $-0.28 \%$ & \\
\hline Annual return & $20.01 \%$ & $10.34 \%$ & $9.00 \%$ & $6.10 \%$ & 0.7 \\
\hline Sharpe ratio & 1.85 & 1.01 & 1.83 & 0.98 & \\
\hline Maximum Drawdown & $10.26 \%$ & $8.65 \%$ & $2.04 \%$ & $1.71 \%$ & \\
\hline
\end{tabular}




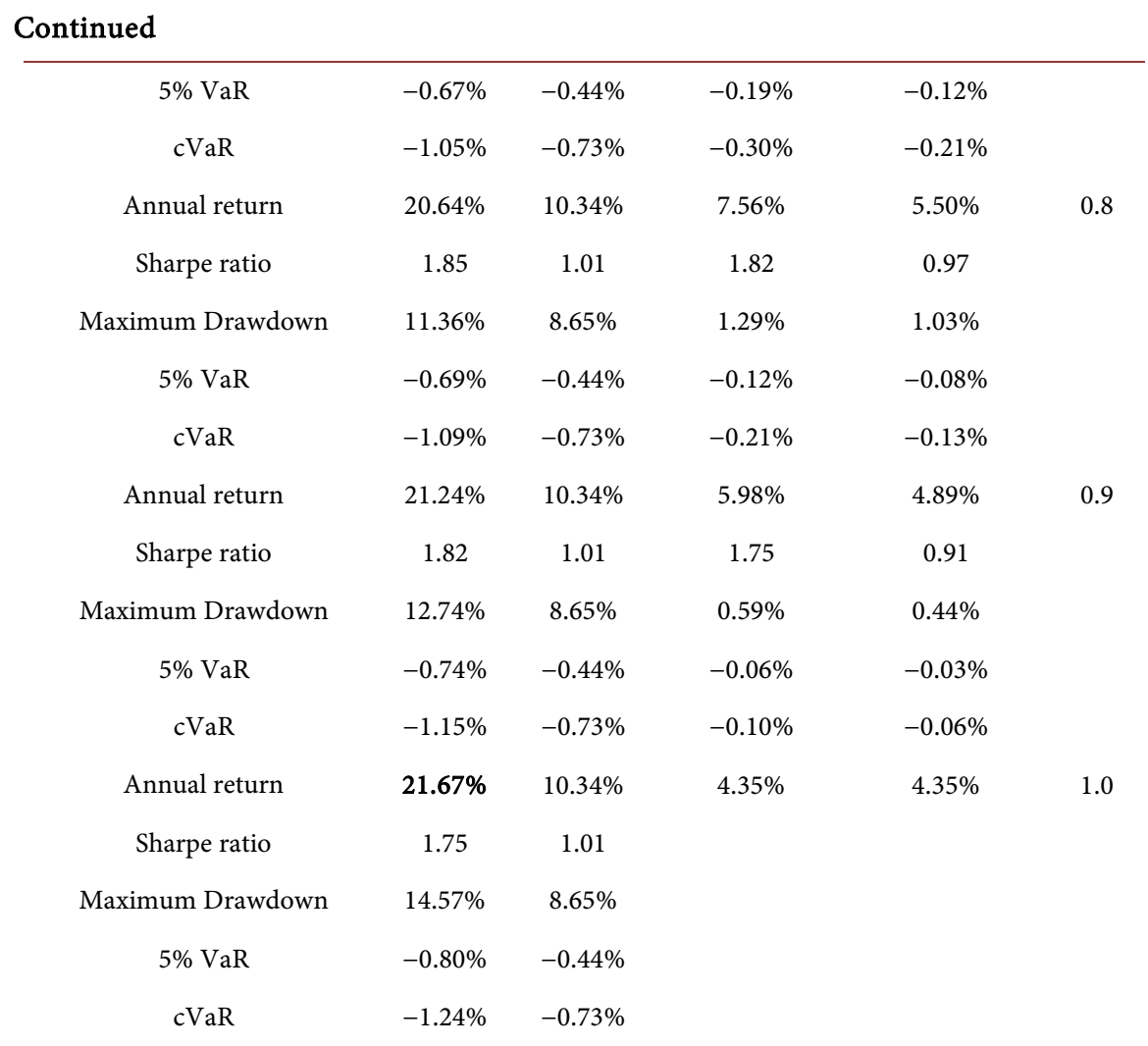

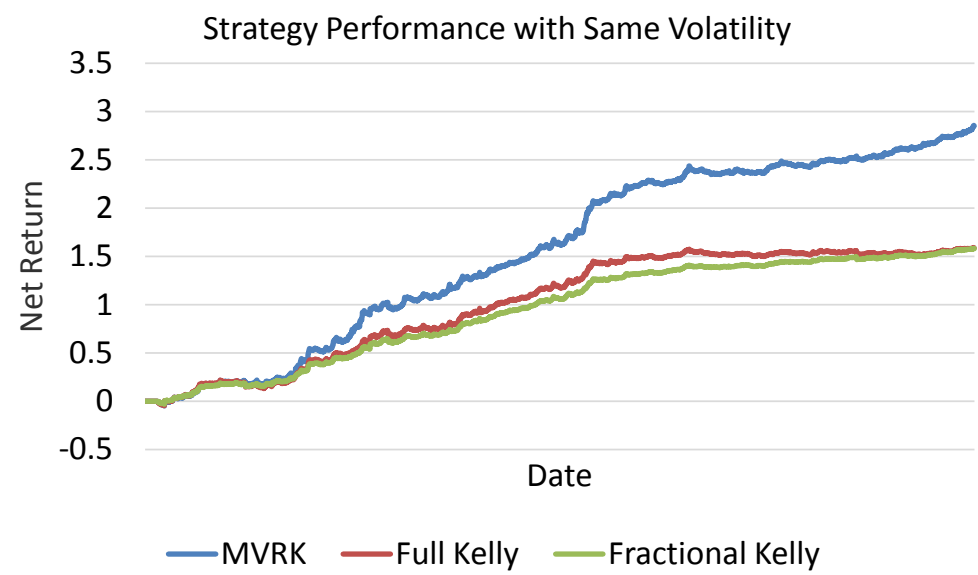

Figure 3. Plots of cumulative net return to the MVRK, Full Kelly and Fractional Kelly strategies. An equal volatility target is applied in three strategies.

\section{Conclusion}

This paper proposes a modified Kelly strategy called Multivariate Volatility Regulated Kelly that outperforms the full Kelly and fractional Kelly strategies in low correlation scenarios. We claim that combining covariance and variance estimators will result in better Kelly strategies in low correlated portfolios. The simulation results show the superiority of MVRK in low correlation settings. The Chinese commodity market further provides empirical evidence of MVRK, and we believe that the global futures market can obtain a similar result. Covariance estimator is time varying and not always stationary. The future work will 
focus on stationary and non-stationary covariance, and analyse the market features when there are change points happening in covariance.

\section{References}

[1] Kelly, J. (1956) A New Interpretation of Information Rate. Bell System Technical Journal, 35, 917-926. https://doi.org/10.1002/j.1538-7305.1956.tb03809.x

[2] Latane, H. (1959) Criteria for Choice among Risky Ventures. Journal of Political Economy, 67, 144-155. https://doi.org/10.1086/258157

[3] Breiman, L. (1961) Optimal Gambling Systems for Favorable Games. Proceedings of the 4 th Berkeley Symposium on Mathematical Statistics and Probability, 1, 63-68.

[4] Thorp, E.O. (1971) Portfolio Choice and the Kelly Criterion. Proceedings of the Business and Economics Section of the American Statistical Association, 215-224.

[5] Hakansson, N.H. (1970) Optimal Investment and Consumption Strategies under Risk for a Class of Utility Functions. Econometrica, 38, 587-607.

https://doi.org/10.2307/1912196

[6] Browne, S. (1997) Survival and Growth with a Liability: Optimal Portfolio Strategies in Continuous Time. Mathematics of Operations Research, 22, 468-493.

https://doi.org/10.1287/moor.22.2.468

[7] Maclean, L.C., Ziemba, W.T. and Blazenko, G. (1992) Growth versus Security in Dynamic Investment Analysis. Management Science, 38, 1562-1585.

https://doi.org/10.1287/mnsc.38.11.1562

[8] Maclean, L.C., et al. (2004) Capital Growth with Security. Journal of Economic Dynamic and Control, 28, 937-954.

https://doi.org/10.1016/S0165-1889(03)00056-3

[9] Maclean, L., Thorp, E. and Ziemba, W.T. (2010) Long-Term Capital Growth: The Good and Bad Properties of the Kelly Criterion. Quantative Finance, 10, 681-687. https://doi.org/10.1080/14697688.2010.506108

[10] Ziemba, W.T. (2003) The Stochastic Programming Approach to Asset, Liability, and Wealth Management. Research Foundation Publication, CFA Institute, AIMR, Charlottesville, VA.

[11] Thorp, E. (2006) The Kelly Criterion in Blackjack, Sports Betting and the Stock Market. Handbook of Asset and Liability Management, North Holland, 1, 385-428. https://doi.org/10.1016/S1872-0978(06)01009-X

[12] Merton, R.C. (1971) Optimal Consumption and Portfolio Rules in a Continuous Time Model. Journal of Economic Theory, 3, 373-413.

https://doi.org/10.1016/0022-0531(71)90038-X

[13] Maclean, L., Ziemba, W.T. and Li, Y. (2005) Time to Wealth Goals in Capital Accumulation and the Optimal Trade-off of Growth versus Security. Quantitative Finance, 5, 343-357. https://doi.org/10.1080/14697680500149552

[14] Davis, M. and Lleo, S. (2010) Fractional Kelly Strategies for Benchmarked Asset Management.

https://www.researchgate.net/publication/228860076_Fractional_Kelly_Strategies_f or_Benchmarked_Asset_Management

[15] MacLean, L.C. and Ziemba, W.T. (2012) Fractional Kelly Strategies in Continuous Time: Recent Developments. In: Davis, M. and Lleo, S., Eds., Handbook of the Fundamentals of Financial Decision Making, World Scientific Publishing, 753-788.

[16] Moskowitz, T.J., Ooi, Y.H. and Pedersen, L.H. (2012) Time Series Momentum. Journal of Financial Economics, 104, 228-250. https://doi.org/10.1016/j.jfineco.2011.11.003 
[17] Ledoit, O. and Wolf, M. (2004) Honey, I Shrunk the Sample Covariance Matrix. Journal of Portfolio Management, 30, 110-119. https://doi.org/10.3905/jpm.2004.110

[18] Shanghai Institute of Futures and Derivatives (2016) The 2016 Development Report on China's Futures Market. Shanghai.

Submit or recommend next manuscript to SCIRP and we will provide best service for you:

Accepting pre-submission inquiries through Email, Facebook, LinkedIn, Twitter, etc. A wide selection of journals (inclusive of 9 subjects, more than 200 journals)

Providing 24-hour high-quality service

User-friendly online submission system

Fair and swift peer-review system

Efficient typesetting and proofreading procedure

Display of the result of downloads and visits, as well as the number of cited articles Maximum dissemination of your research work

Submit your manuscript at: http://papersubmission.scirp.org/

Or contact tel@scirp.org 